\title{
EXACT TIME DEPENDENT CALCULATIONS FOR FLUIDS AND SOLIDS
}

\author{
EDWARD J. KANSA \\ Convergent Solutions, USA
}

\section{ABSTRACT}

Meshfree transport calculations have been traditionally performed in the Lagrangian or particle velocity mode connected to an underlying distorting mesh. That is remapped (interpolated) onto a regular mesh. This primitive procedure was necessary with extremely primitive electronic computers that has extremely restrictive memory storage and execution speed. However, the meshless radial basis function approach allows points to move; without diffusive re-interpolation; one can choose to allow the motion at each interior point to convert the partial differential equation into an exact differential, thereby eliminating truncation errors. Given the proper tools to utilize extended arithmetic precision on computers, electronic computers can asymptotically approach the power of ideal academic mathematics and solve numerically problems approaching theoretical methods.

Keywords: hyperbolic partial differential equations, radial basis functions, exact solutions of time expansion coefficients, exponential convergence, meshless methods.

\section{INTRODUCTION}

Hyperbolic partial differential equations (PDEs) are modified using well-established local mesh-based methods by the introduction of upwind differencing to prevent destabilizing truncation errors. The typical excuse for justifying the modification of the physics is that "in the limit", the artificial unphysical numerical diffusion vanishes. However, on a finite computer, this limit is never reached. This paper demonstrates a numerical meshfree scheme valid for gas, liquid, and solid mechanics that allows for transport without unphysical numerical diffusion by utilizing modern computational methods.

Consider a set of inviscid gas dynamic hyperbolic equations:

$$
\begin{gathered}
\partial \rho / \partial \mathrm{t}+\nabla \bullet \mathrm{m}=0, \\
\partial \mathrm{m} / \partial \mathrm{t}+\nabla \bullet(\mathrm{mm} / \rho+\mathrm{pI})=0, \\
\partial \mathrm{E} / \partial \mathrm{t}+\nabla \bullet((\mathrm{m} / \rho) *(\mathrm{p}+\mathrm{E}))=0,
\end{gathered}
$$

where $\mathrm{m}=\rho u, \rho=$ gas density, $u$ is the velocity vector, $m$, is the momentum density vector, $p$ as the ideal gas pressure, $\mathrm{e}$ is the internal energy, $\mathrm{mm}$ is a $2 \mathrm{nd}$ order momentum dyadic, $\mathrm{I}$ is 2nd order dyadic, $\mathrm{E}=(\rho \mathrm{e}+\mathrm{p}), \gamma=\mathrm{c}_{\mathrm{p}} / \mathrm{c}_{\mathrm{v}}$.

Although gas dynamic equations are primarily longitudinal, i.e. the compressions and rarefactions are in the direction of the rarefactions, in higher dimensions, there is a small amplitude angular momentum wave that propagates in the normal direction of the compression wave.

For elastic solid dynamics, Hooke's law applied to small displacements, u. An ideal solid elastic body can be subject to both compressive and shear stress given by a tensor, $\sigma$.

The conservation of mass is:

$$
\partial \rho / \partial \mathrm{t}+\nabla \bullet(\rho \mathrm{u})=0
$$

The conservation of momentum is now in the form of Hooke's law:

$$
\partial^{2}(\rho \mathbf{u}) / \partial \mathrm{t}^{2}+\nabla^{2} \boldsymbol{\sigma}=0 .
$$


Two dimensional elastic solutions allow for longitudinal waves and transverse waves. Longitudinal waves produce compressions and rarefactions in the direction of wave propagation, whereas transverse waves produce compressions and rarefactions normal to the wave propagation direction.

\section{NUMERICAL SIMULATION OF HYPERBOLIC WAVES}

It must be emphasized here that the mathematics executed on electronic computers is not the same as the mathematics practiced in academia because academic mathematics is non-material existing in the ideal Platonic world in which all rational and irrational numbers have infinite precision, and all transcendental functions are complete to infinite order whereas in computer mathematics, rational and irrational numbers are designated by a finite number of bits comprising a computer word and all transcendental functions are expanded as truncated series expansions or rational fractions. In other words, Plato's description of shadows flickering on a cave wall is most appropriate for mathematics on electronic computers.

Most applied computational engineers, physicists, and mathematicians still use the techniques that were absolutely necessary in early days of electronic computers that hade very limited memory and were extremely slow. Because of severe memory restrictions, it was assumed that the unknown solutions to partial differential and integral equations can be constructed as simple piece-wise constant, linear, or quadratic polynomials.

Kansa [1] showed that the usual derivative expressions of finite differences are really related to a linear or quadric interpolation fit of two or three dependent variables at two or three independent locations. In practice, derivative approximations are limited to quadratic polynomials because derivative approximations are plagued by polynomial snaking.

The next step upward are radial basis functions that depends only upon the geodesic between pairs of points in any geometry. A radial basis function, (RBF), $\phi(x ; y)$, is a function of the geodesic distance, $\mathrm{d}_{\mathrm{g}}$, in Rieman geometry from a data center, $\mathrm{y}$, to an evaluation center, $\mathrm{x}$, where $\mathrm{x}, \mathrm{y} \in \mathfrak{R}^{\mathrm{n}}$. RBFs can be: 1.Polynomials of $\mathrm{d}_{\mathrm{g}}$ having compact support over a limited range, 2. A polynomial with global support, or 3. A transcendental function, continuously differentiable, $\left(\mathrm{C}^{\infty}\right)$, with global support. The $\mathrm{C}^{\infty} \mathrm{RBF}$ s with global support are known theoretically to converge exponentially fast. Madych and Nelson [2], [3] showed that for interpolation, $\mathrm{C}^{\infty} \mathrm{RBF}$ converge as:

$$
\theta^{(<\mathrm{c}>/<\mathrm{h}>)}, \quad \theta<1,
$$

where $<\mathrm{c}>$ is the average shape parameter, $<\mathrm{h}>$ is the average geodesic distance. Note that in most applications of $\mathrm{C}^{\infty} \mathrm{RBFs}$ use the Euclidean radial distance between pairs of points. It was shown in Kansa [4] that partial derivative approximations with $\mathrm{C}^{\infty} \mathrm{RBFs}$ are very accurate negating the need for the weak formulation of finite elements. Because of the accurate approximation of partial derivative, Kansa [4] applied the $\mathrm{C}^{\infty} \mathrm{RBFs}$ to the solution of hyperbolic, parabolic, and elliptic PDEs.

While the $\mathrm{C}^{\infty} \mathrm{RBFs}$ have impressive theoretical properties such as exponential convergence rates, in practice, these RBFs can become so ill-conditioned that the solution is worthless. This phenomenon was explored in Kansa and Holoborodko [5] as being caused by the finite size of the computer words tied to the machine epsilon, $\varepsilon$. Computer chip manufacturers have extremely small demand for computer chips with extended precision, hence chips have 64 or 128 bits. The most prevalent way to obtain extended precision at present is by means of software methods (see www.Advanpix.com). 
Because of the possibility of either severely ill-conditioned or even numerically singular linear equations using single or double precision computers, many researchers were afraid to use $\mathrm{C} \infty \mathrm{RBFs}$ even though the vast amount of domain decomposition literature was available to counter-act ill-conditioning as well as extended precision software.

\section{ELIMINATING DESTABILIZING TRUNCATION ERRORS}

Lagrangian methods have been used extensively to control finite difference, element, and volume truncation errors by allowing mesh points to move at each local convective velocity, $\mathrm{u}_{\mathrm{j}}$. After the mesh points are advected and the local mesh becomes severely distorted, even to the point of having unphysical negative volumes, the mesh is interpolated onto new locations that are not severely distorted. The operation of "remapping" introduces unphysical numerical diffusion, so this process is used sparingly. This is yet another example in which physics is sacrificed for obsolete numerics.

Kansa et al. [6], Kansa [7] and Kansa et al. [8] used meshless $\mathrm{C}^{\infty} \mathrm{RBFs}$ for inviscid Euler equations. In the fixed earth or Eulerian frame, the conservation equations have the form:

$$
\begin{gathered}
\partial \mathrm{U} / \partial \mathrm{t}+\nabla \bullet \mathrm{F}=\mathrm{f}, \quad \text { over } \Omega \backslash \partial \Omega, \\
\mathrm{U} \text { specified on } \partial \Omega,
\end{gathered}
$$

where $\mathrm{F}$ is the appropriate flux vector, and $\mathrm{f}$ is the forcing function.

However, if each data center velocity, $\eta$, is chosen to make each PDE into an exact differential,

$$
\partial \mathrm{U} / \partial \mathrm{t}+\eta \bullet \nabla \mathrm{U}=0, \quad \text { over } \Omega \backslash \partial \Omega,
$$

$\mathrm{U}$ specified on $\partial \Omega$,

where each moving velocity is chosen so that

$$
\begin{gathered}
\{\mathrm{F} \square+\eta \bullet \nabla \mathrm{U}\}=0, \\
\text { along each } \mathrm{dx}_{\mathrm{j}} / \mathrm{dt}=\eta .
\end{gathered}
$$

Although data centers and evaluation points have the freedom to move, this motion must be constrained to prevent points from leaving the domain and any interpolation onto new centers must be constrained to conserve total mass, momentum components, and total energy. Strict conservation is enforced volumetrically by integrating the basis over the evaluation centers.

In the moving frame over $\Omega \backslash \partial \Omega$, it is assumed that space and time are separable. Then the time dependent expansion coefficients over the interior forms a set of time dependent ordinary different equations that have exact solutions.

An excellent numerical test of the theoretical discussion present above is the ideal gas self-similar rarefaction fan. As long as the rarefaction fan is freely propagating without any interactions with solid walls, shocks, contact surfaces, or other rarefaction fans, the sample problem is obtained from Torro's book [9], as well as the physics. This rarefaction fan will have the same shape and will be invariant in magnitude, but not position. A rarefaction wave, in primitive variables, is presented in Tables 1 and 2 at a start time and an ending time that has propagated 1e4 time steps.

The two parts of the table are identical, as they should be if there are negligibly small truncation errors contaminating the solution. This attests to the "exact" nature of the time marching scheme at least to the 43 rd decimal place. 
Table 1: $\quad$ Profile of left rarefaction wave at every fifth data center using the multiquadric radial basis function and extended precision package $(50$ digits $)$ from www.Advanpix.com.

\begin{tabular}{|l|c|c|c|c|c|}
\hline \multicolumn{7}{|c|}{ Time $=0.12425000$} \\
\hline $\begin{array}{l}\text { Gas } \\
\text { density }(\rho)\end{array}$ & $\begin{array}{c}\text { Gas } \\
\text { velocity } \\
(\mathrm{U})\end{array}$ & $\begin{array}{c}\text { Gas } \\
\text { pressure } \\
(\mathrm{P})\end{array}$ & $\begin{array}{c}\text { Left } \\
\text { characteristic } \\
(\mathrm{u}-\mathrm{c})\end{array}$ & $\begin{array}{c}\text { Gas } \\
\text { density } \\
(\mathrm{R})\end{array}$ & $\begin{array}{c}\text { Gas } \\
\text { velocity } \\
(\mathrm{x})\end{array}$ \\
\hline 1.000000 & 0.000000 & 1.000000 & -1.183216 & 5.916080 & 1.615159 \\
\hline 0.830353 & 0.215926 & 0.770846 & -0.924105 & 5.916080 & 1.699435 \\
\hline 0.684553 & 0.185243 & 0.588261 & -0.665994 & 5.916080 & 1.783711 \\
\hline 0.559993 & 0.647779 & 0.444075 & $=0.405992$ & 5.916080 & 1.867987 \\
\hline 0.047759 & 0.971668 & 0.284816 & -0.017215 & 5.916080 & 1.997848 \\
\hline
\end{tabular}

All the eigenvalues are equal to 1.00000000 .

Table 2: $\quad$ Profile of left rarefaction wave at every fifth data center using the multiquadric radial basis function and extended precision package $(50$ digits $)$ from www.Advanpix.com and final time 2.3750000 .

\begin{tabular}{|l|c|c|c|c|c|}
\hline \multicolumn{7}{|c|}{ Time $=2.3750000$} \\
\hline$\rho$ & $\mathrm{U}$ & $\mathrm{P}$ & $\mathrm{u}-\mathrm{c}$ & $\mathrm{R}^{-}$ & $\mathrm{x}$ \\
\hline 1.000000 & 0.000000 & 1.000000 & -1.183216 & 5.916080 & 0.810138 \\
\hline 0.830353 & 0.215926 & 0.770846 & -0.924105 & 5.916080 & -0.194748 \\
\hline 0.684553 & 0.185243 & 0.588261 & -0.665994 & 5.916080 & 1.783711 \\
\hline 0.559993 & 0.647779 & 0.444075 & $=0.405992$ & 5.916080 & 1.867987 \\
\hline 0.047759 & 0.971668 & 0.284816 & -0.017215 & 5.916080 & 1.959115 \\
\hline
\end{tabular}

All the eigenvalues are equal to 1.00000000 .

The variable $\mathrm{R}^{-}$is the left Riemann invariant, for all corresponding values of $\mathrm{u}$ and $\mathrm{c}$.

$$
\mathrm{R}^{-}=\mathrm{u}+2 \mathrm{c} /(\gamma-1)
$$

\section{DISCUSSION}

The mathematics practiced at universities is not the same as the mathematics exercised on electronic computers because all numbers on computers have finite prescribed precision and all functions, especially transcendental functions, are truncated expansions. When extended precision is activated on electronic computers, not only are the word sizes increased, but more software terms are added to library functions, especially for transcendental functions. Persons are familiar with infinite Fourier series expansions for various functions. Although sines and cosines are transcendental functions, these are primarily applied to one-dimensional expansion problems. In addition, such expansions are used for one-dimensional functions without the benefit of any explicit wavelet properties.

The entire class of transcendental functions can be made into $\mathrm{C}^{\infty} \mathrm{RBFs}$ by using either the Euclidean or geodesic distances instead of a point in $\mathfrak{R}^{\mathrm{n}} . \mathrm{C}^{\infty} \mathrm{RBF}$ is much more flexible in application than a transcendental function. Theoretically, in the Platonic ideal world, $\mathrm{C}^{\infty}$ RBFs in interpolation and approximation context, converge exponentially fast as $\theta^{(<\mathrm{c}\rangle /<\mathrm{h}>)}, \theta<$ 1. This exponential convergence rate can be accelerated by a finer discretization or by increasing the average shape parameter, c. Using a finer discretization increases the total number of points, whereas increasing the shape parameter does not, but has the effect of 
increasing the condition number. Whenever the condition number exceeds the inverse of the computer machine epsilon, the round-off errors can render any solution meaningless. However, by decreasing the machine epsilon by increasing the number of active digits by either hardware or software methods enables computers to approach asymptotically the ideal computer.

For time dependent problems, the dependent variable, $\mathrm{U}(\mathrm{x}, \mathrm{t})$ is written as an expansion of spatial $C^{\infty}$ RBFs and products of unknown time dependent expansion coefficients, $\alpha(t)$, allowing each interior point to move at velocity at which the transformed PDE is transformed into an exact differential. The result is that the ODE in the expansion coefficients in the moving frame have exact solutions, and any errors are due to the truncated functions stored in memory. For strictly hyperbolic PDEs, the Riemann solvers, see Toro [9] are recommended for interactions of shock and contact surface discontinuities that produce new waves. For the continuous rarefaction waves, it must be remembered that the function is continuous at the endpoints of the rarefaction, but the derivatives are discontinuous in order to obtain the correct physics. It was demonstrated that a non-interacting rarefaction wave is self-similar, only the data centers expanded; the density, gas velocity, pressure, the characteristic velocity, and the Riemann invariant should be invariant in time, at least to very high order round-off errors, since no computer can compute exactly.

Using more powerful spatial interpolation and approximation schemes without the constraints of local mesh stencils allows researchers to exploit improved software methods that asymptotically approach ideal Platonic academic mathematics.

\section{REFERENCES}

[1] Kansa, E.J., Multiquadrics - A scattered data approximation scheme with applications to computational fluid dynamics: I. Surface approximations and partial derivative estimates. Comput. Math. Appl., 19(6-8), pp. 127-145, 1990.

[2] Madych, W.R. \& Nelson, S.A., Multivariate interpolation and conditionally positive definite functions, II. Math. Comput., 54, pp. 211-230, 1990.

[3] Madych, W.R. \& Nelson, S.A., Bounds on multivariate polynomials and exponential error estimates for multiquadric interpolation. J. Approx. Theory, 70, pp. 94-114, 1992.

[4] Kansa, E.J., Multiquadrics - A scattered data approximation scheme with applications to computational fluid dynamics: II. Solutions to parabolic, hyperbolic, and elliptic partial differential equations. Comput. Math. Appl., 19(6-8), pp. 147-161, 1990.

[5] Kansa, E.J. \& Holoborodko, P., On the ill-conditioned nature of C $\propto-$ RBF strong collocation. Eng. Anal. Bound. Elem., 78, pp. 26-30, 2017.

[6] Kansa, E.J., Power, H. Fasshauer, G.E. \& Ling, L., A volumetric integral radial basis function method for time-dependent partial differential equations: I. Formulation. Eng. Anal. Bound. Elem., 28, pp. 1191-1206, 2004.

[7] Kansa, E.J., Exact explicit time integration of hyperbolic partial differential equations with radial basis functions. Eng. Anal. Bound. Elem., 31, pp. 577-585, 2007.

[8] Kansa, E.J., Aldredge, R.C. \& Ling, L., Numerical simulation of two-dimensional combustion using mesh free methods. Eng, Anal. Bound. Elem., 33, pp. 940-950, 2009.

[9] Toro, E.F., Riemann Solvers and Numerical Methods for Fluid Mechanics, 2nd ed., Springer: Berlin, 1999. 